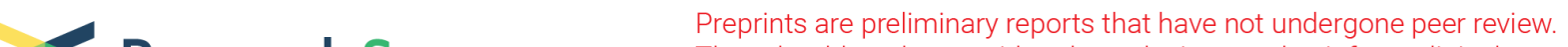

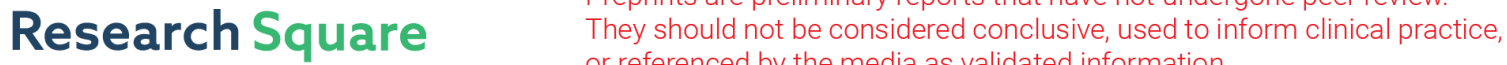 or referenced by the media as validated information.
}

\section{Discoidin Domain Receptor 1 Promotes Hepatocellular Carcinoma Progression through Modulation of SLC1A5 Expression}

\section{Yonglong Pan}

Tongji Hospital of Tongji Medical College of Huazhong University of Science and Technology Mengzhen Han

Tongji Hospital of Tongji Medical College of Huazhong University of Science and Technology Xiaochao Zhang

Tongji Hospital of Tongji Medical College of Huazhong University of Science and Technology Yi He

Tongji Hospital of Tongji Medical College of Huazhong University of Science and Technology

\section{Chaoyi Yuan}

Tongji Hospital of Tongji Medical College of Huazhong University of Science and Technology

\section{Yixiao Xiong}

Tongji Hospital of Tongji Medical College of Huazhong University of Science and Technology

\section{Xinxin Li}

Tongji Hospital of Tongji Medical College of Huazhong University of Science and Technology

\section{Chenglong Zeng}

Tongji Hospital of Tongji Medical College of Huazhong University of Science and Technology

\section{Kan Lu}

Tongji Hospital of Tongji Medical College of Huazhong University of Science and Technology He Zhu

Tongji Hospital of Tongji Medical College of Huazhong University of Science and Technology

\section{Xun Lu}

Tongji Hospital of Tongji Medical College of Huazhong University of Science and Technology

Qiumeng Liu

Tongji Hospital of Tongji Medical College of Huazhong University of Science and Technology

Huifang Liang

Tongji Hospital of Tongji Medical College of Huazhong University of Science and Technology

\section{Zhibin Liao}

Tongji Hospital of Tongji Medical College of Huazhong University of Science and Technology

\section{Zhanguo Zhan}

Tongji Hospital of Tongji Medical College of Huazhong University of Science and Technology

\section{Zeyang Ding}


Tongji Hospital of Tongji Medical College of Huazhong University of Science and Technology

\section{Wanguang Zhang}

Tongji Hospital of Tongji Medical College of Huazhong University of Science and Technology

\section{Bixiang Zhang}

Tongji Hospital of Tongji Medical College of Huazhong University of Science and Technology

Xiao-ping Chen ( $\nabla$ chenxpchenxp@163.com )

Tongji Hospital, Tongji Medical College, Huazhong University of Science and Technology

\section{Research Article}

Keywords: Hepatocellular carcinoma (HCC), Discoid protein domain receptors 1 (DDR1), overall survival (OS), SLC1A5

Posted Date: May 7th, 2021

DOl: https://doi.org/10.21203/rs.3.rs-437636/v1

License: (c) (i) This work is licensed under a Creative Commons Attribution 4.0 International License. Read Full License 


\section{Abstract \\ Background}

Hepatocellular carcinoma (HCC) is one of the most common cancers with the highest mortality rate in the world. Receptor tyrosine kinases play an important role in the occurrence and development of cancer. Discoid protein domain receptors 1 (DDR1) are a special type of transmembrane receptor tyrosine kinase. Here, we show DDR1 is significantly increased in Hepatocellular carcinoma(HCC), which is related to poor prognosis of HCC patients.

\section{Methods}

DDR1 expression in HCC cell lines and paired HCC specimens were determined by western blot and immunohistochemistry (IHC). At the same time, the correlation between the DDR1 and SLC1A5 was also studied in HCC specimens. Cell proliferation ability were evaluated by CCK8 and colony formation assays. Knock-down and overexpression experiments, $\mathrm{CHX}, \mathrm{NH}_{4} \mathrm{CL}$ and $\mathrm{Mg} 132$ intervention experiments, Immunoprecipitation experiments, and nude mouse xenograft models were used to determine the potential mechanism by which DDR1 promotes tumorigenesis in vitro and in vivo.

\section{Results}

In this study, we find that overexpression of DDR1 promotes the proliferation of HCC cells and accelerates the growth of tumor xenografts, while downregulation of DDR1 has the opposite effect. We also proved that loss or gain of DDR1 could affect the cell cycle progression of liver cancer cells. Mechanistically, DDR1 interacts with the SLC1A5 that belongs to the solute carrier (SLC) family of transporters and regulates its stability, thereby regulating the $\mathrm{MTORC} 1$ signaling pathway. In addition, we found that the regulation of SLC1A5 by DDR1 can be restored by lysosome inhibitors. Otherwise, we found that DDR1 was highly expressed in HCC and elevated DDR1 expression predicted shorter overall survival (OS) time for HCC patients. We further revealed that the expression of SLC1A5 was positively correlated with DDR1.In summary, our data shows that DDR1 is a tumor-promoting factor that can control cell proliferation and cell cycle by stabilizing SLC1A5 in a lysosome-dependent pathway, which provides a new treatment target for HCC.

\section{Conclusions}

This study reveals a new mechanism through which DDR1 can play the role of cancer-promoting genes in the progression of liver cancer. We also found that the expression of DDR1 is an independent prognostic indicator of OS, and the expression of DDR1 and SLC1A5 are positively correlated in clinical samples. Our 
findings provide a new perspective for understanding the development of HCC, which may provide new targets for the treatment and management of this challenging cancer.

\section{Background}

Hepatocellular carcinoma is one of the most common types of cancer in the world, and its incidence and fatality rate are among the highest among all cancers. Especially in male patients, the fatality rate is second only to lung cancer, ranking second[1].In China, its incidence is the third highest, and its fatality rate ranks second. Surgery is currently the main method of radical treatment of liver cancer, but the prognosis is not good. Therefore, finding a method of molecular targeted therapy has become our focus of our HCC prevention and treatment[2].The extracellular matrix strictly controls the behavior of cancer cells by inducing multiple signal pathways through cell membrane receptors[3].Among these receptors, receptor tyrosine kinase is a special receptor that plays an important role. DDR1 is one of the receptor tyrosine kinases, and collagen is its natural specific ligand. Collagen can bind and increase kinase activity[4]. DDR1 is activated by various collagens including type I, IV, V, VI, and VIII[5, 6]. Structurally, DDR1 has seven domains, and different domains perform different functions. DDR1 Arg-105 and Ser-175 are essential for high-affinity collagen binding. Amino acids 610-905 are kinase domain, which is closely related to its kinase activity $[7,8]$.Physiologically, DDR1 is expressed mainly limited to epithelial cells, which is located in several organs including skin, kidney, liver and lung. Increased expression of DDR1 has been documented in several types of fibrotic conditions including skin hypertrophic scars, idiopathic pulmonary fibrosis, cirrhotic liver and renal fibrosis[9] .In addition to fibrosis, DDR1 has also been found to be highly expressed in a variety of cancers, including colorectal cancer, breast cancer, oral cancer, lung cancer, thyroid cancer, glioma cancer and esophageal cancer[10-16] .The role of DDR1 in tumor cell survival/proliferation and their crosstalk with other cancer-promoting signaling pathways have been evaluated by some teams in vitro and in vivo. DDR1 is directly regulated by P53 transcription and functions in a feedforward loop to increase p53 levels and at least some of its effectors. However Inhibition of DDR1 function leads to a significant increase in apoptosis of p53 wild-type cells[17] .In the glioma cell lines and pancreatic adenocarcinoma cell lines, siRNA against DDR1 can significantly suppress cell proliferation and invasion $[18,19]$.After DDR1 is silenced, the upregulation of transforming growth factor $\beta 1$ (TGF $\beta 1$ ) is thought to induce tumor cell growth arrest[19]. In addition, DDR1 plays a vital role at least partially through the functional interaction with Notch1[20] .In KRAS-driven lung adenocarcinoma, combined inhibition of targeting DDR1 and Notch signaling is an extremely effective treatment strategy[8]. Further, complement $\mathrm{C} 1 \mathrm{q}$ stimulates the progression of hepatocellular tumors through the activation of DDR1[21] .Moreover, non-coding RNA is also involved in the regulation of DDR1. The miR-199a-3p targets the 3'UTR of DDR1 mRNA to reduce DDR1 expression. The circ-NSD2 promotes the expression of DDR1 by down-regulating miR-199b-5p[22, 23] .

Previous studies have found that DDR1 is up-regulated in liver cancer tissues and is closely related to liver cancer recurrence[24] .However, the mechanism of how DDR1 affects the occurrence and development of liver cancer is not particularly clear. Here we focus on the relationship between DDR1 and SLC1A5. We found that DDR1 combines with SLC1A5. SLC1A5, also called ASCT2, is a neutral amino 
acid transporter belonging to the SLC1 family, located in the plasma membrane of several body parts. In addition, the latest study found that a variant of SLC1A5 can be distributed on the mitochondrial membrane. It is a mitochondrial glutamine transporter that plays a role in the metabolic reprogramming of cancer cells[25].SLC1A5 is found to be highly expressed in a variety of cancer tissues and promote the proliferation of cancer cells[26-32] .The important proto-oncogene MYC of liver cancer can directly bind to the promoter of SLC1A5 and up-regulate its expression, and the up-regulation of SLC1A5 caused glutamine uptake can also activate the mTORC1 pathway[33-35]. In this study, we proved that DDR1 is highly expressed in liver cancer. DDR1 promotes the proliferation of liver cancer cells by binding to and stabilizing SLC1A5, activating the mTORC1 pathway.

\section{Methods}

Tissue specimens and Immunohistochemistry

A total of 66 paired specimens of tumor and adjacent non-tumor tissues were collected from $66 \mathrm{HCC}$ patients who underwent hepatectomy at the Hepatic Surgery Center, Tongji Hospital of Huazhong University of Science and Technology (HUST) (Wuhan, China). Matched fresh specimens of HCC tissues and adjacent non-tumorous liver tissue were lysed separately for western blot analysis. A tissue microarray of 110 pairs of primary HCC tissues with their clinical and prognosis data were acquired from the specimen library of the Hepatic Surgery Center, Tongji Hospital of Huazhong University of Science and Technology. The samples were obtained in surgeries from 2012/2/16 to 2014/4/1, and the database has been updated every month since 2012/2/16. All HCC tissues were identified in Tongji hospital's department of pathology. Before surgery, there was no local or systemic therapy. After surgery, no anticancer treatment was conducted before recurrence. Written informed consent was obtained from all patients and each procedure was approved by the Ethical Committee of Tongji Hospital. The fundamental processes of the Immunohistochemistry assay have been mentioned previously[45]. The image scores were evaluated by 3 different pathologists ignorant of patient clinical pathological characteristics. The total score of each image was calculated by multiplying the score of staining area percentage by intensity score as mentioned previously[45]. The total scores of $\geq 5$ was defined as positive; Otherwise, defined as negative.

\section{Cell lines and cell culture}

The lentivirus packaging cell line 293T, human fetal liver cell line HL-7702, hepatoma cell line HepG2, HCC cell lines Hep3B, Huh7, SK-Hep1, HLF, HLE, HCCLM3, PLC/PRF/5, 97H and Bel7402 were purchased from China Center for Type Culture Collection (CCTCC, Wuhan, China). The cell lines were cultivated in Dulbecco's Modified Eagle's Medium (DMEM, Gibco, ThermoFisher Scientific, Waltham, Massachusetts, USA) supplemented with $10 \%$ fetal bovine serum (FBS, Gibco, North America) at $37^{\circ} \mathrm{C}$ in $5 \% \mathrm{CO} 2$ and $95 \%$ air.

Reagents and Antibodies 
Rat tail Collagen I (BD Bioscience, MA, USA), DDR1 inhibitor imatinib and ponatinib (MedChemExpress, NJ, USA). Puromycin, trypsin-EDTA, Opti-MEM medium and polybrene were obtained as previous described[45]. Lipofectamine 2000 Reagent and Lipofectamine 3000 Reagent were purchased from Invitrogen (Life Technologies, Carlsbad, CA, USA). CHX CQ and Mg132 were obtained from MedChemExpress (Shanghai, China). NH4Cl was obtained from promoter company (promoter, Wuhan, China). All antibodies used in the project were detailed in Supporting Table S1.

Plasmid construction and small interference RNAs

The human DDR1 (NM_001954.4) cDNA, and pcDNA3.1 plasmid were gifts from the Hepatic Surgery Center, Tongji Hospital, Huazhong University of Science and Technology, P .R. China. pBABE-puro (Plasmid \#1764), gag/pol (Plasmid \#14887), pMD2.G(Plasmid \#12259), pLK0.1-TRC cloning vector (Plasmid \# 10878), psPAX2(Plasmid \#12260) were purchased from Addgene (Addgene, Cambridge, MA, USA). To establish pBABE-Flag-DDR1 or pBABE-Flag-SLC1A5 plasmid, the human CDNA was cloned into the BamHI/EcoRI site of the pBABE-puro retroviral vector, and was identified by sequencing (TSINGKE, Wuhan, China). To construct pLKO.1-scramble, pLK0.1-shDDR1, and pLK0.1-shSLC1A5, the target doublestranded oligonucleotides (shRNA) sequences and one non-targeting sequence (negative control, scramble) were annealed and cloned into the Agel/EcoRI site of pLKO.1 vector. The sequences of target shRNA oligo pairs are listed in Supporting Table S2. Viral production, infection, establishment of stable cell clones were described previously[46]. pcDNA3.1 plasmid inserted by Flag-, Myc- or HA- tagged DDR1 and its mutants, Flag- or HA-tagged SLC1A5, Flag-tagged PPP2R1A, SRFBP1 and CUL4B were constructed according to ClonExpress II One Step Cloning Kit and Mut Express II Fast Mutagenesis Kit V2 (Vazyme, Nanjing, China) protocol and were identified by sequencing (TSINGKE, Wuhan, China).

Cell growth assay

Sk-hep1-DDR1 ( $1.5^{*} 10^{3}$ cells/ well), HepG2-DDR1 ( $1.5^{*} 10^{3}$ cells/ well), HLF-shDDR1 ( $1 * 10^{3}$ cells/well), HLE-shDDR1 ( $1 * 10^{3}$ cells/well), Sk-hep1-SLC1A5 (1.5*10 ${ }^{3}$ cells/ well), HepG2-SLC1A5( $1.5^{\star} 10^{3}$ cells/well), Hep3B-shSLC1A5( $2 * 10^{3}$ cells/well), PLC/PRF/5-shSLC1A5(2*103 cells/ well) and the same amount of their control cells were cultured in 96-well plate. At the indicated time points, the Cell Counting Kit-8 (CCK8 , Beyotime Institute of Biotechnology) was added to the cells for 2 hours, and then the plate was read using an enzyme-linked immunosorbent assay plate reader (Bio-Tek Elx 800, USA) at $450 \mathrm{~nm}$. Cells (500 cells/well) were plated in each well of a 6 -well plate. At day 14 the plates were fixed in $4 \%$ paraformaldehyde, stained with $1 \%$ crystal violet, and the numbers of colonies greater than $100 \mu \mathrm{m}$ in diameter were counted and analyzed by Image $\mathrm{J} 1.52 \mathrm{a}(\mathrm{NIH}, \mathrm{USA})$.

Cell cycle analysis

For cell cycle analysis, the cells were plated in a 6-well culture plate and grown for 24 hours. Then incubate the cells with $1 \mathrm{mM}$ thymidine(Sigma-Aldrich) for 24 hours to synchronize the cells at the G1/S or $\mathrm{G} 2 / \mathrm{M}$ boundary. The cells were then treated with fresh medium containing $2.5 \mathrm{lg} / \mathrm{mL} \mathrm{TM}$ for different lengths of time. Next, the cells were trypsinized, washed twice with cold PBS, and fixed with cold 70\% 
ethanol overnight at $20^{\circ} \mathrm{C}$. The cells were then washed twice with PBS and incubated with a PBS solution of $10 \mathrm{mg} / \mathrm{mL}$ RNase $A, 400 \mathrm{mg} / \mathrm{mL}$ propidium iodide, and $0.1 \%$ Triton $X$ at room temperature (RT) for 30 minutes. The cells were then analyzed by flow cytometry.

Immunoprecipitation and western blotting

Immunoblotting assay and co-immunoprecipitation assay were performed as described previously. [46]Briefly, cells were collected and lysed on ice with IP lysis buffer. lysates were incubated with protein $G$ agarose for 2 hours, and immunoprecipitated with indicated antibodies at $4^{\circ} \mathrm{C}$ overnight. Then lysates were incubated with protein $\mathrm{G}$ agarose for 1 hours followed by 1 wash using IP lysis buffer and 3 washes using washing buffer (300 mM NaCl, 1.0 mM EDTA, $25 \mathrm{mM}$ Tris-HCl, pH7.4, 1.0\% NP-40). The beads were eluted with 2×SDS-PAGE loading buffer and then subjected to immunoblotting analysis.

Reverse transcription PCR and Real-time quantitative PCR

Total cell RNA was extracted with TRIzol Reagent (Invitrogen, Life Technologies, Carlsbad, CA, USA). Reverse transcription was carried out with the QuantScript RT Kit (TIANGEN, Beijing, China) according to manufacturers' introductions. Real-time fluorescence quantitative PCR was performed with the CFX96 Touch $^{\text {TM }}$ Real-Time PCR Detection System (Bio-Rad, Hercules, CA, USA) using SuperReal PreMix Plus (SYBR Green) kit (TIANGEN, Beijing, China) according to the manufacturer's protocol (Each gene expression level was normalized to that of glyceraldehyde-3-phosphate dehydrogenase (GAPDH) of the same sample. Each sample was done in triplicate independently. The primers are listed in Supporting Table S3.

Mass spectrometry

293T cells transiently transfected with FLAG-DDR1 or FLAG-vector were lysed in IP lysis buffer (25mM Tris- $\mathrm{HCl}$ (pH 7.4), $150 \mathrm{mM} \mathrm{NaCl}, 1 \%$ NP-40, $1 \mathrm{mM}$ EDTA, $10 \%$ Glycerol and protease inhibitor cocktails) and IP assays were performed as described previously[46]. The eluted proteins were separated by SDS-PAGE and then sent to mass spectrometry for detection. Mass spectrometry was detected and analyzed by ptmbio lab (PTM BIO, Hangzhou, China).

Immunofluorescence

Immunofluorescence assay was performed as described previously[46]. In brief, after the indicated treatments, cells were cultured on coverslips for $12 \mathrm{~h}$, fixed in $4 \%$ paraformaldehyde for $15 \mathrm{~min}$ at room temperature, and permeabilized with $0.5 \%$ Triton X-100 for 20 min. After blocking, the slides were incubated with according primary antibody overnight at $4^{\circ} \mathrm{C}$ in a humidified box. After that, the slides were then washed three times and incubated with according secondary antibody for $4 \mathrm{~h}$ at room temperature in a humidified box. Finally, cell nuclei were stained by 40,60-diamidino-2-phenylindole (DAPI, Sigma-Aldrich) for $5 \mathrm{~min}$. Pictures were obtained by phase-contrast and confocal laser-scanning microscopy. 
Subcutaneous xenograft study in nude mice

Four-week-old male BALB/c (nu/nu) mice were raised under specific pathogen-free conditions. All animals were cared for according to the Guide for the Care and Use of Laboratory Animals. The experimental protocol was approved by the Committee on the Ethics of Animal Experiments of the Tongji Medical College, HUST. Mice were subcutaneously injected with $4 \times 10^{6}$ cells and tumor size was measured every 4 days. The volume of tumor was calculated by the formula: volume $=0.52 \times$ (long diameter) $\times$ (short diameter)/2. Tumors were excised at 30 days post injection followed by photograph and weigh. After that, tumors were fixed with $4 \%$ paraformaldehyde and applied to immunohistochemical analysis.

Statistics analysis

Data analysis was performed using Prism 6.0 (GraphPad Software, La Jolla, CA, USA) software. The results were presented as the mean \pm SEM. The difference between two groups were analyzed by twotailed Student's t-test, ANOVA test, or a nonparametric test. $\chi 2$ test or Fisher's exact test was used to analyze categorical data. Association between DDR1 and SLC1A5 expression in HCC tissues was calculated using Pearson correlation test. The survival between subgroups was assessed by KaplanMeier and log-rank analysis. Three independent experiments were carried out to insure repeatability. A value of $P<0.05$ was regarded as statistically significance.

\section{Results}

High expression of DDR1 is associated with poor prognosis in HCC patients.

In order to check whether DDR1 plays an important role in liver cancer, we used Western Blotting (WB) to detect the protein levels of DDR1 in 66 pairs of human liver cancer tissue and their matched non-tumor liver samples (Fig. 1A). Western blot showed that in HCC tissues, the protein level of DDR1 increased compared to its matched non-tumor specimens (Fig. 1B). These results indicate that DDR1 is upregulated in liver cancer and may play an important role in tumor development. Since the expression of DDR1 is upregulated in HCC tumors, we next investigate the clinical significance of DDR1 in HCC patients. First, we analyzed the expression of DDR1 by performing immunohistochemical staining on a microarray containing 110 pairs of human liver cancer and surrounding non-tumor tissues (Fig. 1C). Compared with matched non-tumor tissues, the expression level of DDR1 was significantly increased in HCC tissues (Fig. 1D). More importantly, Kaplan-Meier analysis showed that the high expression of DDR1 in liver cancer is related to the deterioration of OS in HCC patients (Fig. 1E). The results from the KM-plotter HCC cohort demonstrated that HCC patients in the group with high DDR1 expression exhibited shorter overall survival than that exhibited by the low-expression group (log-rank, $p=0.026$ ), as determined using Kaplan-Meier analysis (Fig. 1F). In summary, these results indicate that the expression of DDR1 is upregulated in HCC, and DDR1 may be a valuable biomarker in HCC patients.

DDR1 promotes the proliferation and G1-S phase transition in HCC cells 
In order to evaluate the effect of DDR1 on the proliferation of liver cancer cells, we constructed stable overexpression or knockdown cell lines of DDR1 based on the expression level of DDR1 in some liver cancer cell lines (Supplementary Fig. S1A). We use the constructed retrovirus to overexpress DDR1 stably in Sk-hep1 and HepG2 cell lines, and we use the constructed lentivirus to knock down DDR1 stably in HLF and HLE cell lines (Supplementary Fig. S1B-E). we performed cck8 assays and colony formation assays. Sk-hep1 or HepG2 cells overexpressing DDR1 showed increased proliferation compared with that of control cells, and they also formed $\sim 2$-fold more colonies than did the control cells (Fig. 2A-B and Supplementary Fig. S1F-G). At the same time, we determined the cell-cycle distributions of SK-hep1 cells by flow cytometry. Our results showed that the overexpression of DDR1 increased the proportion of cells entering the $S$ phase and decreased the proportion of cells entering the G1 phase (Fig. 2C-D), indicating that the overexpression of DDR1 induced G1-S phase transition in HCC cells. To further validate whether DDR1 can promote the proliferation of hepatocellular carcinoma cells in vivo, a xenograft tumor model in nude mice was employed. The HCC cells (Sk-hep1) stably overexpressing DDR1 were subcutaneously injected into nude mice. The tumorigenic efficiency of each group of cancer cells was evaluated 27 days after injection. SK-hep1 cells overexpressing exogenous DDR1 formed tumors of 3.5-fold larger size than those in the control group (Fig. 2E-F). These data further indicated that DDR1 promotes cell proliferation in HCC. Conversely, knockdown of DDR1 in HLF and HLE cells reduced cell proliferation and formed $40 \%$ or even fewer clones compared with that of the control cells (Fig. 2G-H and Supplementary Fig. S1H-I). Similarly, we determined the cell-cycle distributions of HLF cells by flow cytometry. Our results showed that knockdown of DDR1 reduced the proportion of cells entering the $S$ phase and increased the proportion of cells entering the $\mathrm{G} 1$ phase (Fig. 2l-J). We employed xenograft tumor models in nude mice to find HLF cells with endogenous knockdown of DDR1 formed tumors about $32 \%$ smaller than the controls (Fig. 2K-L), which again suggested an oncogenic effect of DDR1 protein. In summary, these results indicate that DDR1 improve the proliferation of $\mathrm{HCC}$ and cell cycle progression.

DDR1 interacts with SLC1A5 and promotes proliferation through SLC1A5.

To investigate how DDR1 promote proliferation in HCC cells, we overexpressed Flag-tagged DDR1 in HEK293T(293T). Then, we performed an immunoprecipitation mass spectrometry (IP-MS) using anti-flag antibody, and the peptides corresponding to lots of protein (such as PPP2R1A, SEFBP1, SLC1A5 and CUL4B) were identified (Fig. 3A-B and Supplementary Fig. S2A). To verify the IP-MS outcome, we respectively co-transfected 293T cells with Myc-tagged DDR1 and Flag-tagged PPP2R1A, SEFBP1, SLC1A5 and CUL4B in 293T cells and performed IP with Flag antibody. Among these candidates, SLC1A5 was selected because of its powerful interaction with DDR1(Supplementary Fig. S2B). We further confirmed the interaction by exogenous Co-IP assay, which Flag-tagged SLC1A5 and HA-tagged DDR1 were transiently transfected into 293T cells (Fig. 3C). Furthermore, this interaction was also found by endogenous Co-IP assay in HLF and Sk-hep1 (Fig. 3D). The colocalization of these two proteins was further confirmed by confocal laser measurement both in 293T and HLF (Fig. 3E). We found that the interaction happens on the cell membrane. In order to find the exact domains of DDR1 responsible for binding with SLC1A5, we made a series of DDR1 truncation mutations (Fig. 3F). These truncation mutations all preserve transmembrane domain. We co-transfected HA-tagged SLC1A5 and FLAG-tagged 
DDR1 and its truncated mutations in 293T cells, and then performed Co-IP assay with anti-flag antibody. We found that the aa1-455, aa1-609, aa410-609 and aa410-913 truncated mutations of DDR1 all interacts with SLC1A5(Fig. $3 G$ ). These results suggest that the $447-455$ region of DDR1 protein binds to SLC1A5 protein. In order to further understand whether the binding of SLC1A5 to DDR1 is ligand and kinase activity dependent, we adopt two DDR1 functional mutants to perform co-IP with SLC1A5. DDR1 is a receptor tyrosine kinase, so we changed arginine 105 to alanine (R105A) to lower the affinity with the major ligand of DDR1, Collagen $₫$. Similarly, we mutated lysine 618 to alanine (K618A) to inhibit kinase activity of DDR1. We co-transfected two DDR1 mutants and SLC1A5 in 293T, and found that the interaction between SLC1A5 and the two mutants of DDR1 still exists (Supplementary Fig. S2C). This shows that structurally, the two mutants of DDR1 do not affect its binding to SLC1A5. Then we wanted to clarify the role of SLC1A5 in DDR1's tumor-promoting effect. We overexpressed SLC1A5 in HLF-shDDR1 cells, and then found that the effect of suppressing proliferation after knocking down DDR1 was restored. (Fig. $3 \mathrm{H}$ ). The above shows that DDR1 binds to SLC1A5 and exerts an anti-proliferation effect in tumors through SLC1A5.

DDR1 stabilizes SLC1A5 through a kinase-independent manner

We already found that the interaction of DDR1 and SLC1A5 happens on the cell membrane, we next determined whether DDR1 regulates function and status of SLC1A5. Interestingly, SLC1A5 protein expression was evidently up-regulated in DDR1 overexpressed HCC cells (Fig. 4A and Supplementary Fig. S3A). On the contrary, we detected significantly lower levels of SLC1A5 protein expression in HLF and HLE cells with DDR1 knockdown (Fig. 4B and Supplementary Fig. S3B). We tested the tyrosine phosphorylation level of DDR1 after Collagen 『stimulation in Sk-hep1 and HLF cells, and confirmed that Collagen $\otimes$ can indeed promote the activation of DDR1 by increasing tyrosine phosphorylation level of DDR1 ( Supplementary Fig. S3C). However, the positive regulation of DDR1 on SLC1A5 seems to be independent of Collagen \in HCC cells(Fig. 4C and Supplementary Fig. S3D). Furthermore, DDR1 overexpressed SK-hep1 and control cells were pretreated with or without Collagen『alone or in combination with ponatinib and imatinib (DDR1 kinase inhibitor). The effect of DDR1 stabilizing SLC1A5 has not been rescued by DDR1 kinase inhibitor (Fig. 4D). We also did similar experiments on the unmodified HLE and got unanimous conclusions (Supplementary Fig. S3E). At the same time, the downregulation of SLC1A5 by DDR1-knockdown is not time-dependent with Collagen in HLF cells(Fig. 4E). The above results indicate that DDR1 stabilizes the expression of SLC1A5 protein through a kinaseindependent mechanism. In addition, although treatment with $\mathrm{CHX}$ for $12 \mathrm{~h}$ will significantly reduce SLC1A5 levels in Sk-hep1-vector cells, the same treatment method does not significantly affect SLC1A5 levels in Sk-hep1-DDR1 cells (Fig. 4F-G), which indicates that DDR1 may extend the half-life of SLC1A5 by maintaining its stability in Sk-hep1 cells. Then we started to explore how DDR1 stabilizes SLC1A5. We used MG132(inhibitor of proteasome) and $\mathrm{NH}_{4} \mathrm{CL}$ (lysosome inhibitors) to treat SK-hep1 and HLF cells and found that only $\mathrm{NH}_{4} \mathrm{CL}$ could restore the effect of DDR1 in stabilizing SLC1A5 (Fig. $4 \mathrm{H}$ and Supplementary Fig. S3F-G). Therefore, we have reason to believe that DDR1 stabilizing SLC1A5 is achieved through autophagy-lysosomal degradation pathway. Such data indicate that SLC1A5 is 
stabilized by DDR1 in a kinase activity-independent manner. As we all know, SLC1A5 is highly expressed in many cancers, and is also related to the influx of essential amino acids, the mammalian target of rapamycin (mTOR) activation, and L-GIn-dependent tumor cell growth[35, 37] .To determine whether DDR1 can regulate the mTORC1 signaling pathway in HCC cells, we performed Western blot analysis. The results showed that overexpressed DDR1 enhanced the phosphorylation level of mTORC1 and S6K in the SK-hep1 and HepG2 cell lines (Fig. 4I). Similarly, knockdown of DDR1 in HLF and HLE cells reduced the phosphorylation levels of mTORC1 and 4EBP1 (Fig. 4J). At the same time, we knocked down SLC1A5 in SK-hep1 cells overexpressing DDR1, and we found that the activation of $\mathrm{mTORC} 1$ signaling pathway by DDR1 was partially restored (Supplementary Fig. S3H). The above data proves that DDR1 can regulate the mTORC1 signaling pathway through SLC1A5.

SLC1A5 promotes cellular proliferation, and mTORC1 signaling pathway in HCC cells

To evaluate the effect of SLC1A5 on the proliferation of liver cancer cells, we constructed a stable SLC1A5 overexpression or knockdown cell line based on the expression level of SLC1A5 in HCC cell lines (Supplementary Fig. S4A and S4C). We performed cck8 assay and colony formation experiment. Compared with control cells, Sk-hep1 or HepG2 cells expressing SLC1A5 showed stronger proliferation potential (Fig. 5A-D). In contrast, knockdown of SLC1A5 in HCC cells reduced cell proliferation and formed $40 \%$ or fewer clones compared to control cells (Fig. 5E-H). Similarly, we also collected the proteins of the above cells to detect the activity of mTORC1 signaling pathway. We found that the mTORC1 signaling pathway was activated by by SLC1A5 (Fig. $5 \mathrm{I}-\mathrm{J}$ ). These data further indicate that SLC1A5 promotes the proliferation, and mTORC1 signaling pathway of HCC cells.

SLC1A5 is highly expressed in liver cancer and is positively correlated with DDR1.

To examine whether SLC1 A5 has important roles in human liver cancer, we first analyzed the expression level of SLC1A5 in HCC by analyzing RNA-seq data from GEPIA2[38]. The expression levels of SLC1A5 were significantly upregulated in HCC (Fig. 6A). Next, we assessed the protein levels of SLC1A5 in 62 pairs of human HCC tissue samples and their matched nontumorous liver specimens by western blot. The protein level of SLC1A5 was significantly increased in HCC tissues compared with their matched nontumorous liver specimens (Fig. 6B and Supplementary Fig. S4D). To further define the clinical significance of SLC1A5, we analyzed its expression in tissue microarrays containing 110 paired human hepatoma and peripheral nontumor tissues by immunohistochemical staining with anti-SLC1A5 antibody. Compared with matched nontumorous tissues, the expression level of SLC1A5 was significantly increased in HCC tissues (Fig. 6C-D). More importantly, Kaplan-Meier analysis showed that the high expression of SLC1A5 in liver cancer is related to the deterioration of OS in HCC patients. Strikingly, low SLC1A5 expression was significantly associated with an increase in overall survival (Fig. 6E). The results from the TCGA and KM-plotter HCC cohort demonstrated that HCC patients in the group with high SLC1A5 expression exhibited shorter overall survival time than that exhibited by the low-expression group, as determined using Kaplan-Meier analysis (Fig. 6F-G). In order to analyze the correlation between the expression of DDR1 and SLC1A5 in HCC, we performed a correlation analysis on the expression of cancer 
tissue in the tissue microarray. We found that the expression of SLC1A5 and DDR1 has a strong correlation in hepatocellular carcinoma tissues (Fig. $6 \mathrm{H}-\mathrm{J}$ ). Similarly, western blot analysis showed that the protein expression level of SLC1A5 is positively correlated with the expression level of DDR1 in 43 pairs HCC tissue and their matched nontumorous liver specimens (Fig. 6K). These results indicated that the expression of SLC1A5 is up-regulated in HCC expression, and SLC1A5 expression positively correlated with DDR1 in clinical samples.

\section{Discussion}

The carcinogenic effect of DDR1 in human cancer was first highlighted by global phosphoserines profile analysis in lung cancer[39]. Since then, DDR1 has been reported to play a considerable role in a variety of cancers $[11-16,22,40]$. There are also some reports that DDR1 plays a role in liver cancer[21] . But it is not clear what mechanism DDR1 plays in liver cancer. In this study, we provide evidence to support the hypothesis that DDR1 is involved in SLC1A5 post-transcriptional regulation. DDR1 is a special receptor tyrosine kinase, most of which is located on the membrane as the receptor of collagen I and IV. SLC1A5 is an important amino acid transporter and belongs to the solute carried (SLC) family transporters[41, 42] .We found that DDR1 interacts with SLC1A5 and maintains its stability by inhibiting its degradation in lysosome. Collagen $\llbracket$ can promote DDR1 tyrosine phosphorylation when stimulating HCC cells. However,

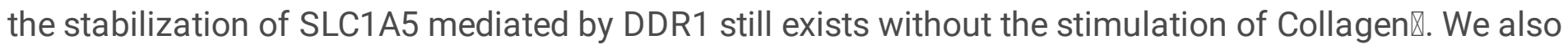
used ponatinib and imatinib, the classic inhibitors of DDR1, and the effect of DDR1 on SLC1A5 has not changed significantly. This indicates that the mechanism by which DDR1 stabilizes SLC1A5 has nothing to do with kinase activity.

SLC1A5 plays an important role in cellular glutamine transport, and the intake of glutamine is very important to maintain the growth of tumor cells[37, 43]. As we know, mTORC1 can sense changes in glutamine in the cell and then respond. Metabolism of glutamine and its entry into the TCA cycle are required for mTORC1 activation[44]. With the deepening of research, we found that DDR1 can regulate the activation of the mTORC1 signaling pathway, causing an increase in the phosphorylation of mTORC1 and the phosphorylation of its downstream substrates S6K and 4EBP1, thereby regulating the proliferation and cell cycle of HCC cells. To prove the correlation between DDR1 and SLC1A5 in tissue samples, we used serial sections of tissue microarray to evaluate the correlation between the expression levels of DDR1 and SLC1A5. Otherwise, we used DDR1 and SLC1A5 antibodies to perform western blot experiments on the proteins extracted from frozen patient samples. These proved the positive correlation between DDR1 and SLC1A5 in protein expression. More importantly, the overall survival time of patients with low SLC1A5 is significantly longer than that of patients with high SLC1A5. Similarly, patients with low DDR1 levels have significantly longer overall survival time than those with high DDR1 levels. In addition, overexpression of DDR1 can promote tumor growth in mouse subcutaneous tumor transplantation models, indicating that DDR1 may be a target for HCC treatment in the future. And the impacts of DDR1 in HCC cells were mediated by SLC1A5. 
After we discovered that the way DDR1 stabilizes SLC1A5 is not dependent on its kinase activity. We are trying to figure out which degradation mode of SLC1A5 was interfered by DDR1. Next, we used the proteasome inhibitor Mg132 and found that it did not restore the regulation of SLC1A5 by DDR1. So, we used another common inhibitor of protein degradation pathway, ammonium chloride. Ammonium chloride is an inhibitor of the lysosomal degradation pathway. We were pleasantly surprised to find that ammonium chloride can restore the stability of DDR1 to SLC1A5. This shows that DDR1's regulation of SLC1A5 is achieved by inhibiting it's lysosomal protein degradation. However, the molecular model through which DDR1 regulates the lysosomal degradation process of SLC1A5 is currently unclear. To figure out the internal mechanism may be our next research direction.

\section{Conclusions}

Overall, our findings indicate that the protein expression of DDR1 in liver cancer is correlated with the protein expression of glutamine transporter SLC1A5, and positively regulates its stability, which ultimately promotes the growth of hepatocellular carcinoma.

\section{Abbreviations}

CCK8

Cell Counting Kit-8;

$\mathrm{CHX}$

Cycloheximide;

OS

Overall survival;

$\mathrm{HCC}$

Hepatocellular carcinoma;

IHC

Immunohistochemistry;

OD

Optical density;

PCR

Polymerase Chain Reaction.

\section{Declarations}

\section{Ethics approval and consent to participate}

All experiments containing patient specimens or data were approved by the Ethics Committee of Tongji Hospital, HUST and were performed in accordance with the Declaration of Helsinki Principles. Prior written and informed consent was obtained from each patient. 


\section{Consent for publication}

All materials and images are original. No consent needs to declare.

\section{Competing interests}

The authors declare no competing interest.

\section{Funding}

This work was supported by the National Natural Science Foundation of China (No. 81572427, $81874189,81572855,81874065$, and 81874149), National Basic Research Program of China (2020YFA0710700), the State Key Project on Infection Disease of China (No. 2018ZX10723204-003) and Tongji Hospital (HUST) Foundation for Excellent Young Scientist (No. 2020YQ05).

\section{Authors' contribution}

$\mathrm{YP}, \mathrm{MH}$ and $\mathrm{XZ}$ conceived and designed the study. $\mathrm{YP}, \mathrm{MH}$ and $\mathrm{XZ}$, performed the experiments. $\mathrm{YH}, \mathrm{CY}, \mathrm{YX}$, $\mathrm{XL}, \mathrm{CZ}$ and $\mathrm{KL}$ collected the clinical specimens and data. YP performed the statistical analysis and wrote the manuscript. $\mathrm{HZ}, \mathrm{XL}, \mathrm{QL}, \mathrm{HL}$ provided technical or material support. ZL, ZZ and ZD gave a lot of guiding opinions. WZ, BZ and XC contributed to the writing and critical reading of the paper. All authors read and approved the final manuscript.

\section{Acknowledgements}

As the first author of this study, I (Yonglong Pan) want to thank my mother, father and my girlfriend.

\section{References}

1. Bray F, Ferlay J, Soerjomataram I, Siegel RL, Torre LA, Jemal A. Global cancer statistics 2018 : GLOBOCAN estimates of incidence and mortality worldwide for 36 cancers in 185 countries. CA Cancer J Clin. 2018;68(6):394-424.

2. Wu J, Yang S, Xu K, et al. Patterns and Trends of Liver Cancer Incidence Rates in Eastern and Southeastern Asian Countries (1983-2007) and Predictions to 2030. Gastroenterology. 2018;154(6):1719-28.e5.

3. Mohan V, Das A, Sagi I. Emerging roles of ECM remodeling processes in cancer. Semin Cancer Biol. 2020;62:192-200.

4. Rammal H, Saby C, Magnien K, et al. Discoidin Domain Receptors: Potential Actors and Targets in Cancer. Front Pharmacol. 2016;7:55.

5. Vogel W. Discoidin domain receptors: structural relations and functional implications. FASEB J. 1999;13 Suppl:77-82. 
6. Nielsen M. Estimation of noradrenaline and its major metabolites synthesized from [3H] tyrosine in the rat brain. J Neurochem. 1976;27(2):493-500.

7. Abdulhussein R, McFadden $C$, Fuentes-Prior P, Vogel WF. Exploring the collagen-binding site of the DDR1 tyrosine kinase receptor. J Biol Chem. 2004;279(30):31462-70.

8. Ambrogio C, Gómez-López G, Falcone M, et al. Combined inhibition of DDR1 and Notch signaling is a therapeutic strategy for KRAS-driven lung adenocarcinoma. Nat Med. 2016;22(3):270-7.

9. Moll S, Desmoulière A, Moeller MJ, et al. DDR1 role in fibrosis and its pharmacological targeting. Biochim Biophys Acta Mol Cell Res. 2019;1866(11):118474.

10. Vella V, Nicolosi ML, Cantafio P, et al. DDR1 regulates thyroid cancer cell differentiation via IGF-2/IR-A autocrine signaling loop. Endocr Relat Cancer. 2019;26(1):197-214.

11. Zhong X, Zhang W, Sun T. DDR1 promotes breast tumor growth by suppressing antitumor immunity. Oncol Rep. 2019;42(6):2844-54.

12. Jeitany $M$, Leroy $C$, Tosti $P$, et al. Inhibition of DDR1-BCR signalling by nilotinib as a new therapeutic strategy for metastatic colorectal cancer. EMBO Mol Med. 2018;10(4).

13. Heinzelmann-Schwarz VA, Gardiner-Garden M, Henshall SM, et al. Overexpression of the cell adhesion molecules DDR1, Claudin 3, and Ep-CAM in metaplastic ovarian epithelium and ovarian cancer. Clin Cancer Res. 2004;10(13):4427-36.

14. Weiner HL, Huang H, Zagzag D, Boyce H, Lichtenbaum R, Ziff EB. Consistent and selective expression of the discoidin domain receptor-1 tyrosine kinase in human brain tumors. Neurosurgery. 2000;47(6):1400-9.

15. Nemoto T, Ohashi K, Akashi T, Johnson JD, Hirokawa K. Overexpression of protein tyrosine kinases in human esophageal cancer. Pathobiology. 1997;65(4):195-203.

16. Valencia K, Ormazábal C, Zandueta C, et al. Inhibition of collagen receptor discoidin domain receptor1 (DDR1) reduces cell survival, homing, and colonization in lung cancer bone metastasis. Clin Cancer Res. 2012;18(4):969-80.

17. Ongusaha PP, Kim JI, Fang L, Wong TW, Yancopoulos GD, Aaronson SA, Lee SW. p53 induction and activation of DDR1 kinase counteract p53-mediated apoptosis and influence p53 regulation through a positive feedback loop. EMBO J. 2003;22(6):1289-301.

18. Yamanaka R, Arao T, Yajima $\mathrm{N}$, et al. Identification of expressed genes characterizing long-term survival in malignant glioma patients. Oncogene. 2006;25(44):5994-6002.

19. Rudra-Ganguly N, Lowe $C$, Mattie $M$, et al. Discoidin domain receptor 1 contributes to tumorigenesis through modulation of TGFBI expression. PLoS One. 2014;9(11):e111515.

20. Kim HG, Hwang SY, Aaronson SA, Mandinova A, Lee SW. DDR1 receptor tyrosine kinase promotes prosurvival pathway through Notch1 activation. J Biol Chem. 2011;286(20):17672-81.

21. Lee JH, Poudel B, Ki HH, Nepali S, Lee YM, Shin JS, Kim DK. Complement C1q stimulates the progression of hepatocellular tumor through the activation of discoidin domain receptor 1 . Sci Rep. 2018;8(1):4908. 
22. Deng Y, Zhao F, Hui L, Li X, Zhang D, Lin W, Chen Z, Ning Y. Suppressing miR-199a-3p by promoter methylation contributes to tumor aggressiveness and cisplatin resistance of ovarian cancer through promoting DDR1 expression. J Ovarian Res. 2017;10(1):50.

23. Chen LY, Zhi Z, Wang L, et al. NSD2 circular RNA promotes metastasis of colorectal cancer by targeting miR-199b-5p-mediated DDR1 and JAG1 signalling. J Pathol. 2019;248(1):103-15.

24. Jian ZX, Sun J, Chen W, Jin HS, Zheng JH, Wu YL. Involvement of discoidin domain 1 receptor in recurrence of hepatocellular carcinoma by genome-wide analysis. Med Oncol. 2012;29(5):3077-82.

25. Yoo HC, Park SJ, Nam M, et al. A Variant of SLC1A5 Is a Mitochondrial Glutamine Transporter for Metabolic Reprogramming in Cancer Cells. Cell Metab. 2020;31(2):267. :- 83.e12..

26. Wang $\mathrm{C}, \mathrm{Wu} \mathrm{J}$, Wang $\mathrm{Z}$, et al. Glutamine addiction activates polyglutamine-based nanocarriers delivering therapeutic siRNAs to orthotopic lung tumor mediated by glutamine transporter SLC1A5. Biomaterials. 2018;183:77-92.

27. van Geldermalsen M, Wang Q, Nagarajah R, et al. ASCT2/SLC1A5 controls glutamine uptake and tumour growth in triple-negative basal-like breast cancer. Oncogene. 2016;35(24):3201-8.

28. Toda K, Nishikawa G, Iwamoto M, Itatani Y, Takahashi R, Sakai Y, Kawada K. Clinical Role of ASCT2 (SLC1A5) in KRAS-Mutated Colorectal Cancer. Int J Mol Sci. 2017;18(8).

29. Csanadi A, Oser A, Aumann K, et al. Overexpression of SLC1a5 in lymph node metastases outperforms assessment in the primary as a negative prognosticator in non-small cell lung cancer. Pathology. 2018;50(3):269-75.

30. Bjersand K, Seidal T, Sundström-Poromaa I, Åkerud H, Skirnisdottir I. The clinical and prognostic correlation of HRNPM and SLC1A5 in pathogenesis and prognosis in epithelial ovarian cancer. PLoS One. 2017;12(6):e0179363.

31. Bröer A, Rahimi F, Bröer S. Deletion of Amino Acid Transporter ASCT2 (SLC1A5) Reveals an Essential Role for Transporters SNAT1 (SLC38A1) and SNAT2 (SLC38A2) to Sustain Glutaminolysis in Cancer Cells. J Biol Chem. 2016;291(25):13194-205.

32. Zhang Z, Liu R, Shuai Y, Huang Y, Jin R, Wang X, Luo J. ASCT2 (SLC1A5)-dependent glutamine uptake is involved in the progression of head and neck squamous cell carcinoma. $\mathrm{Br} \mathrm{J}$ Cancer. 2020;122(1):82-93.

33. Nakaya $M$, Xiao $Y$, Zhou $X$, et al. Inflammatory $T$ cell responses rely on amino acid transporter ASCT2 facilitation of glutamine uptake and mTORC1 kinase activation. Immunity. 2014;40(5):692-705.

34. Liu P, Ge M, Hu J, et al. A functional mammalian target of rapamycin complex 1 signaling is indispensable for c-Myc-driven hepatocarcinogenesis. Hepatology. 2017;66(1):167-81.

35. Nicklin P, Bergman P, Zhang B, et al. Bidirectional transport of amino acids regulates mTOR and autophagy. Cell. 2009;136(3):521-34.

36. Scalise M, Pochini L, Cosco J, Aloe E, Mazza T, Console L, Esposito A, Indiveri C. Interaction of Cholesterol With the Human SLC1A5 (ASCT2): Insights Into Structure/Function Relationships. Front Mol Biosci. 2019;6:110. 
37. Hassanein M, Hoeksema MD, Shiota M, et al. SLC1A5 mediates glutamine transport required for lung cancer cell growth and survival. Clin Cancer Res. 2013;19(3):560-70.

38. Tang Z, Kang B, Li C, Chen T, Zhang Z. GEPIA2: an enhanced web server for large-scale expression profiling and interactive analysis. Nucleic Acids Res. 2019;47(W1):W556-6W560.

39. Rikova K, Guo A, Zeng Q, et al. Global survey of phosphotyrosine signaling identifies oncogenic kinases in lung cancer. Cell. 2007;131(6):1190-203.

40. Chou ST, Peng HY, Mo KC, et al. MicroRNA-486-3p functions as a tumor suppressor in oral cancer by targeting DDR1. J Exp Clin Cancer Res. 2019;38(1):281.

41. Jiang H, Zhang N, Tang T, Feng F, Sun H, Qu W. Target the human Alanine/Serine/Cysteine Transporter 2(ASCT2): Achievement and Future for Novel Cancer Therapy. Pharmacol Res. 2020;158:104844.

42. Lafitte M, Sirvent A, Roche S. Collagen Kinase Receptors as Potential Therapeutic Targets in Metastatic Colon Cancer. Front Oncol. 2020;10:125.

43. Ito K, Suda T. Metabolic requirements for the maintenance of self-renewing stem cells. Nat Rev Mol Cell Biol. 2014;15(4):243-56.

44. Kim SG, Hoffman GR, Poulogiannis G, et al. Metabolic stress controls mTORC1 lysosomal localization and dimerization by regulating the TTT-RUVBL1/2 complex. Mol Cell. 2013;49(1):17285 .

45. Ding ZY, Jin GN, Liang HF, et al. Transforming growth factor $\beta$ induces expression of connective tissue growth factor in hepatic progenitor cells through Smad independent signaling. Cell Signal. 2013;25(10):1981-92.

46. Ding ZY, Jin GN, Wang W, et al. Reduced expression of transcriptional intermediary factor 1 gamma promotes metastasis and indicates poor prognosis of hepatocellular carcinoma. Hepatology. 2014;60(5):1620-36.

\section{Figures}


A

$\begin{array}{lllllllllll}\mathrm{T} 1 & \mathrm{~N} 1 & \mathrm{~T} 2 & \mathrm{~N} 2 & \mathrm{~T} 3 & \mathrm{~N} 3 & \mathrm{~T} 4 & \mathrm{~N} 4 & \mathrm{~T} 5 & \mathrm{~N} 5\end{array}$

DDR1

GAPDH

$\begin{array}{llllllllll}\text { T6 } & \text { N6 } & \text { T7 } & \text { N7 } & \text { T8 } & \text { N8 } & \text { T9 } & \text { N9 } & \text { T10 } & \text { N10 }\end{array}$

DDR1

GAPDH
C

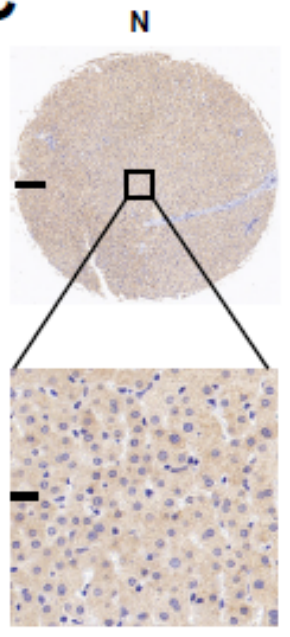

D

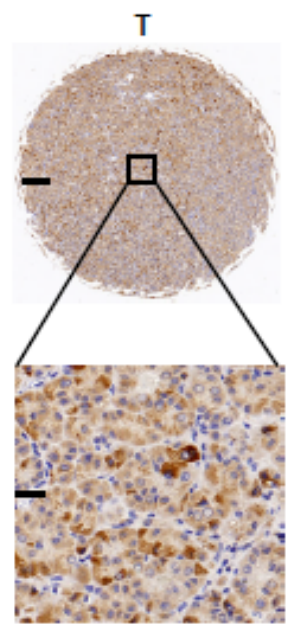

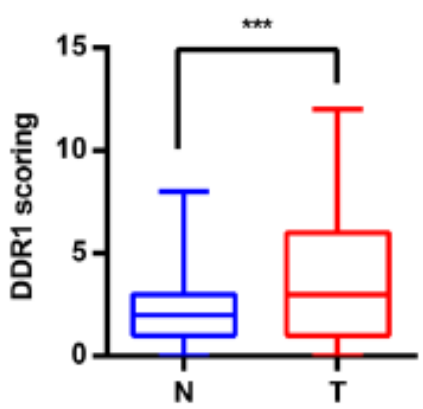

$\mathbf{F}$

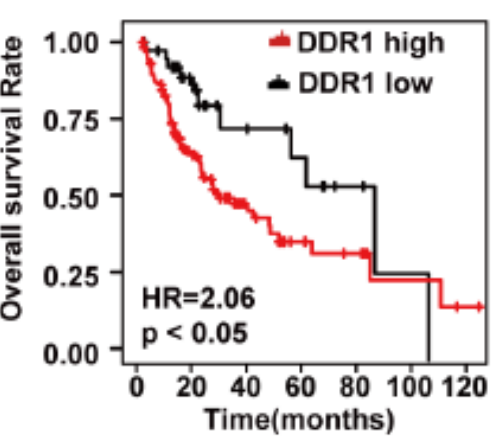

Figure 1

High expression of DDR1 is associated with poor prognosis in HCC patients. $a, b$ The protein level of DDR1 was examined in 66 pairs of HCC tumor tissues (T) and corresponding peritumor tissues (N) by western blot using anti-DDR1 antibody, where GAPDH was used as a loading control. Representative WB images were presented. The relevant density of DDR1 protein was analyzed and is shown. c, d Two representative immunohistochemical stains on a tissue array containing $110 \mathrm{HCC}$ samples with anti DDR1 antibody are shown. The average integrated optical density (IOD) of positive areas was analyzed. Scale bar represents $250 \mu \mathrm{m}$ (up), $25 \mu \mathrm{m}$ (down). e Overall survival analysis of HCC patients with DDR1 expression in 110 samples. f Overall survival analysis of HCC patients with DDR1 expression from the data of KM plotter. Statistics were performed using Student's two-sided $t$ test. ** $P<0.01$. 
A

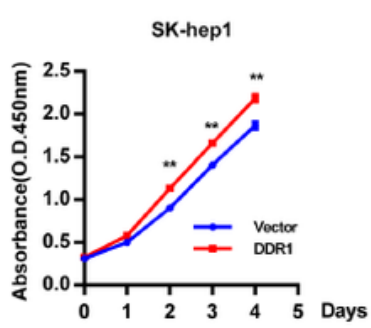

C

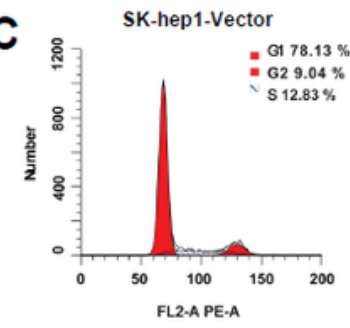

E

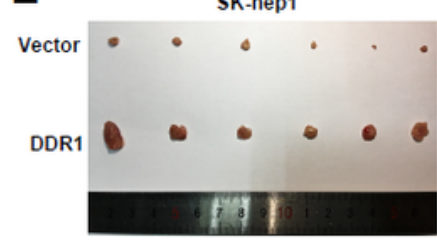

G

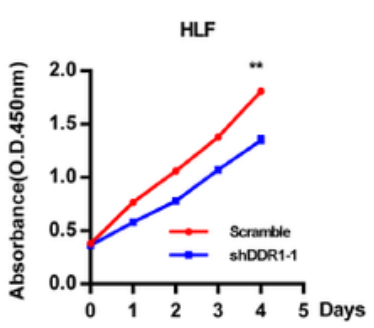

I

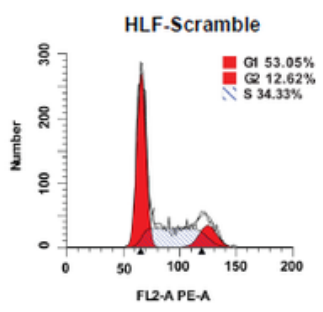

K

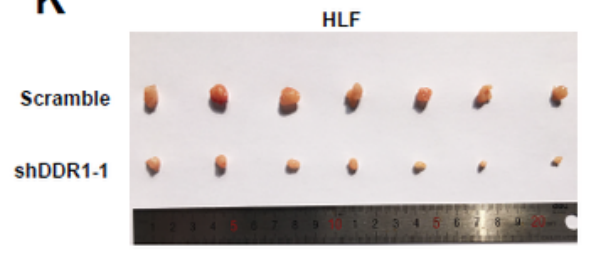

$F$

H
B
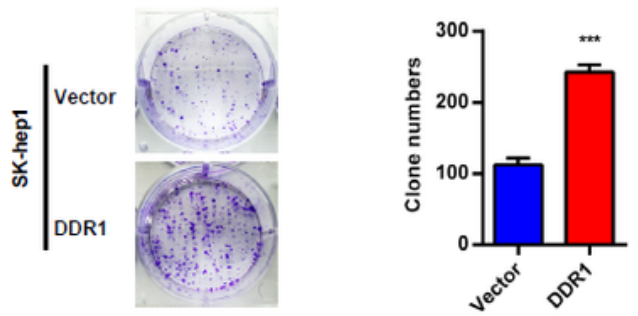

SK-hep1-DDR1

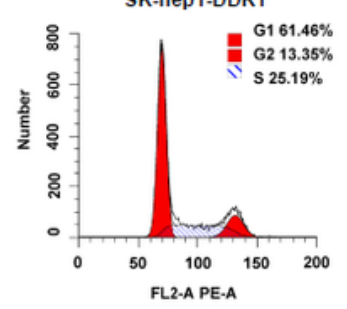

D
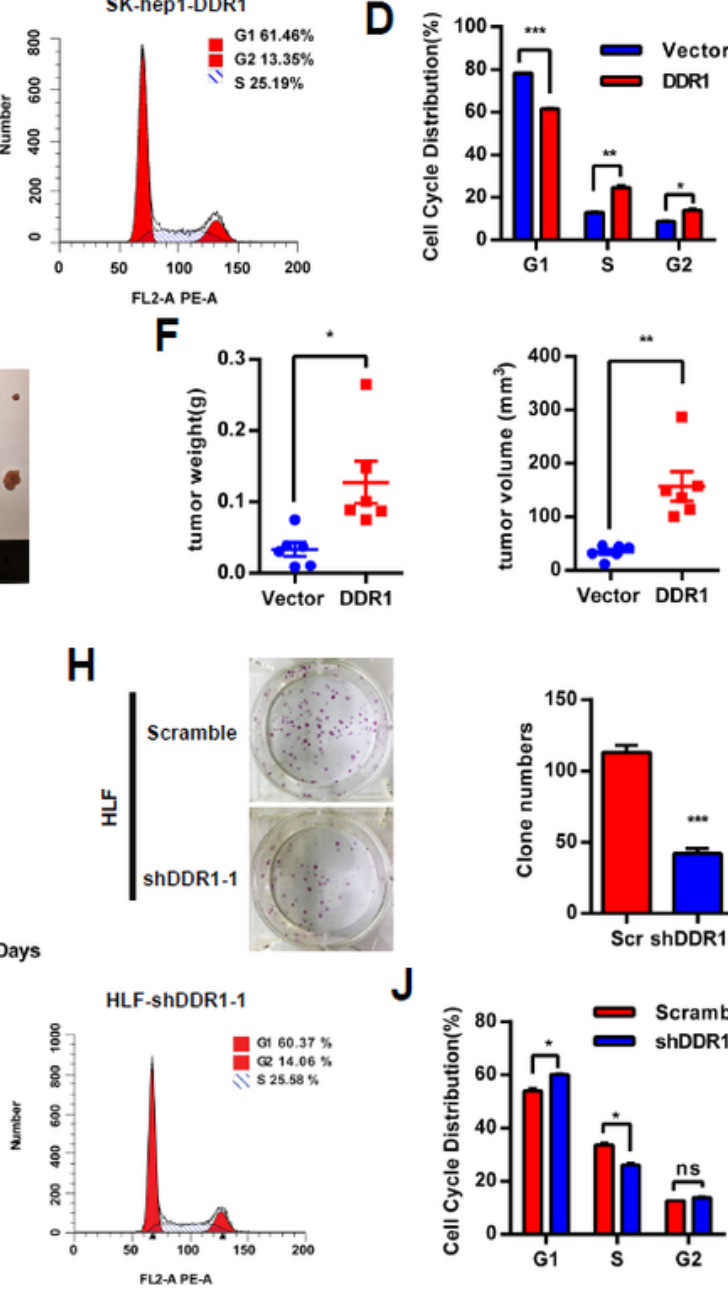

J

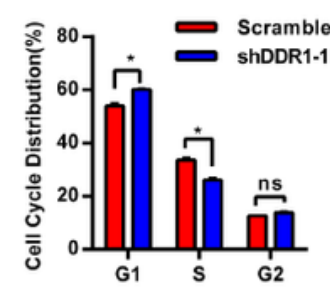

L

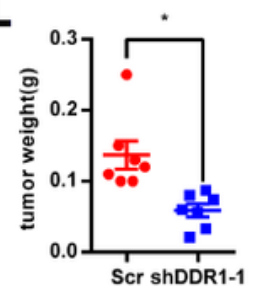

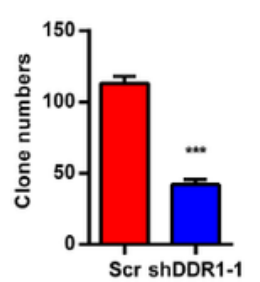

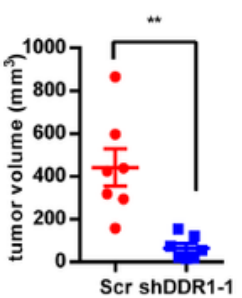

Figure 2

DDR1 promotes the proliferation and G1-S phase transition in HCC cells. a The effect of DDR1 overexpressing on SK-hep1 cell proliferation was assessed by the CCK8 assay. $b$ The effect of DDR1 overexpression on SK-hep1 cell proliferation was assessed by a colony-formation assay. c, d The effect of DDR1 overexpression on cell cycle of SK-hep1 cells was analyzed by flow cytometry. e, $f$ Overexpression of DDR1 promotes the growth of xenograft tumors derived from SK-hep1 cells. The 
growth of xenograft tumors was measured by tumor volume and tumor weight. g CCK8 assay for the indicated cells. $h$ colony-formation assay for the indicated cells. $i, j$ The effect of DDR1 knockdown on cell cycle of HLF cells was analyzed by flow cytometry. k. I Knockdown of DDR1 inhibits the growth of xenograft tumors derived from HLF cells. The growth of xenograft tumors was measured by tumor volume and tumor weight. Statistics were performed using Student's two-sided $t$ test. ${ }^{*} P<0.05, * \star P<$ $0.01, * * * \mathrm{P}<0.001$.

A

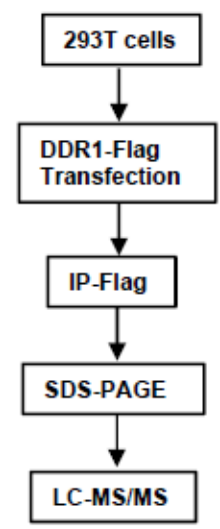

D

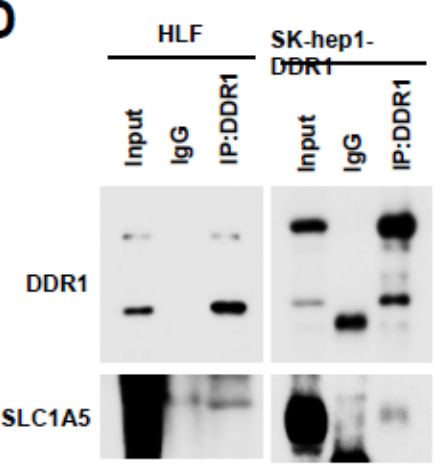

$\mathbf{F}$
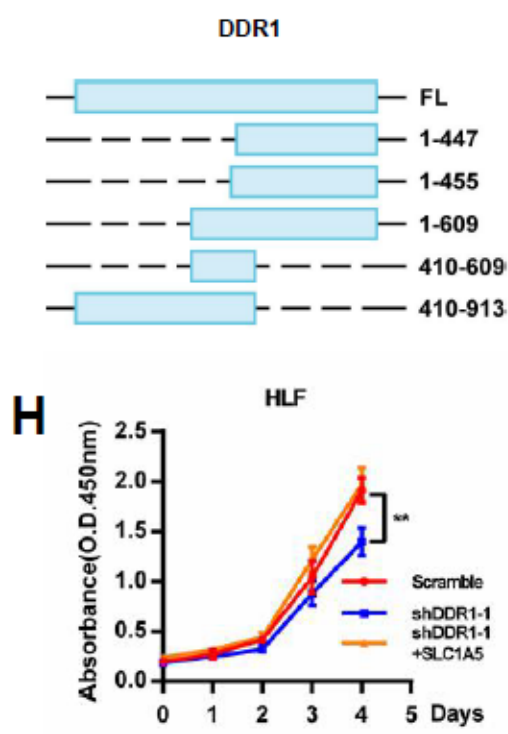

C
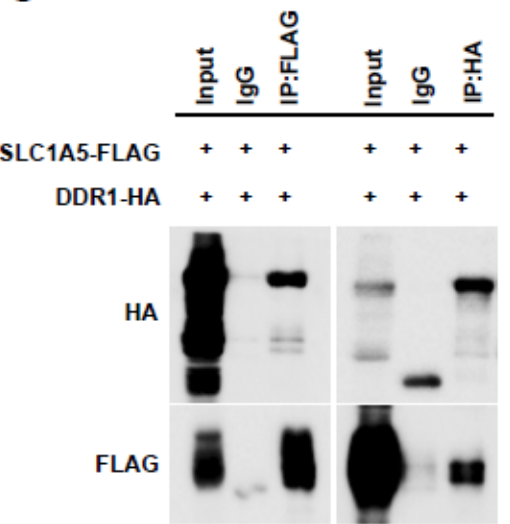

\begin{tabular}{|l|l}
\hline 69.47 & 2 \\
\hline
\end{tabular}

E

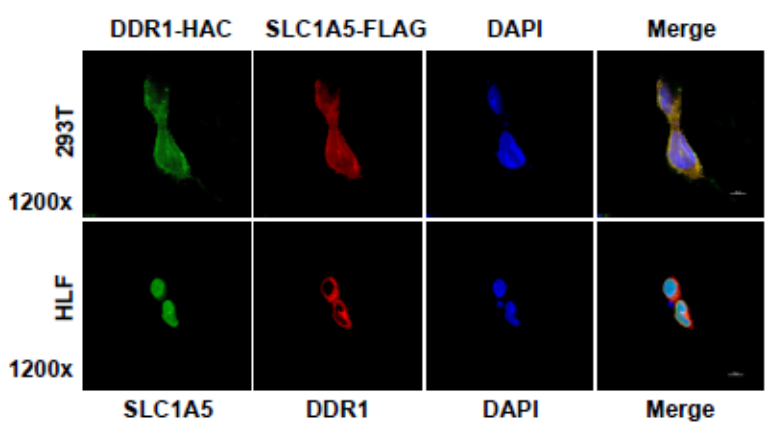

G
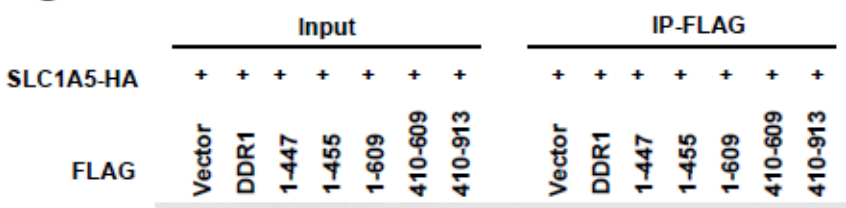

HA

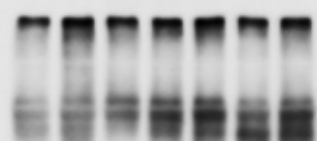

FLAG

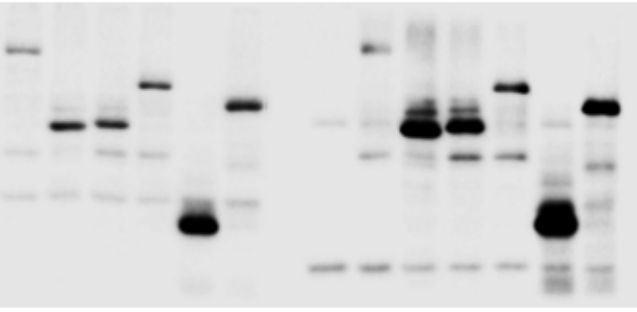

Figure 3 
DDR1 interacts with SLC1A5 and promotes proliferation through SLC1A5. a Cellular extracts from 293T cells transiently transfected with FLAG-tagged DDR1 or FLAG-tagged vector were subsequently immunoprecipitated with anti-FLAG antibody. Eluted proteins were separated on SDS-PAGE and identified by mass spectrometry analysis. $b$ The table describes mass spectrometric results identifying the bait protein DDR1 and its binding partners. c 293T cells were transiently co-transfected with Flag-tagged SLC1A5 and HA-tagged DDR1. d Endogenous SLC1A5 was immunoprecipitated with an anti-DDR1 antibody, and IgG was used as the negative control. Immunocomplexes were analyzed with anti-DDR1 and anti-SLC1A5 antibodies. e Confocal assays was shown to observe the co-localization of exogenously expressed DDR1 and SLC1A5 in 293T cells (Scale bar: 10 $\mu \mathrm{m}$ ). Confocal assays was shown to observe the co-localization of endogenous DDR1 and SLC1A5 in HLF cells (Scale bar: 10 $\mu \mathrm{m}$ ).f Schematic representation of FLAG-tagged full-length DDR1 (FL) and various deletion mutants. g HEK-293T cells were co-transfected with the indicated FLAG-tagged DDR1 and SLC1A5 constructs, and the interaction between DDR1 and SLC1A5 was determined by immunoprecipitation (IP) and immunoblotting (IB). $\mathrm{h}$ CCK8 assay in the indicated cells. 


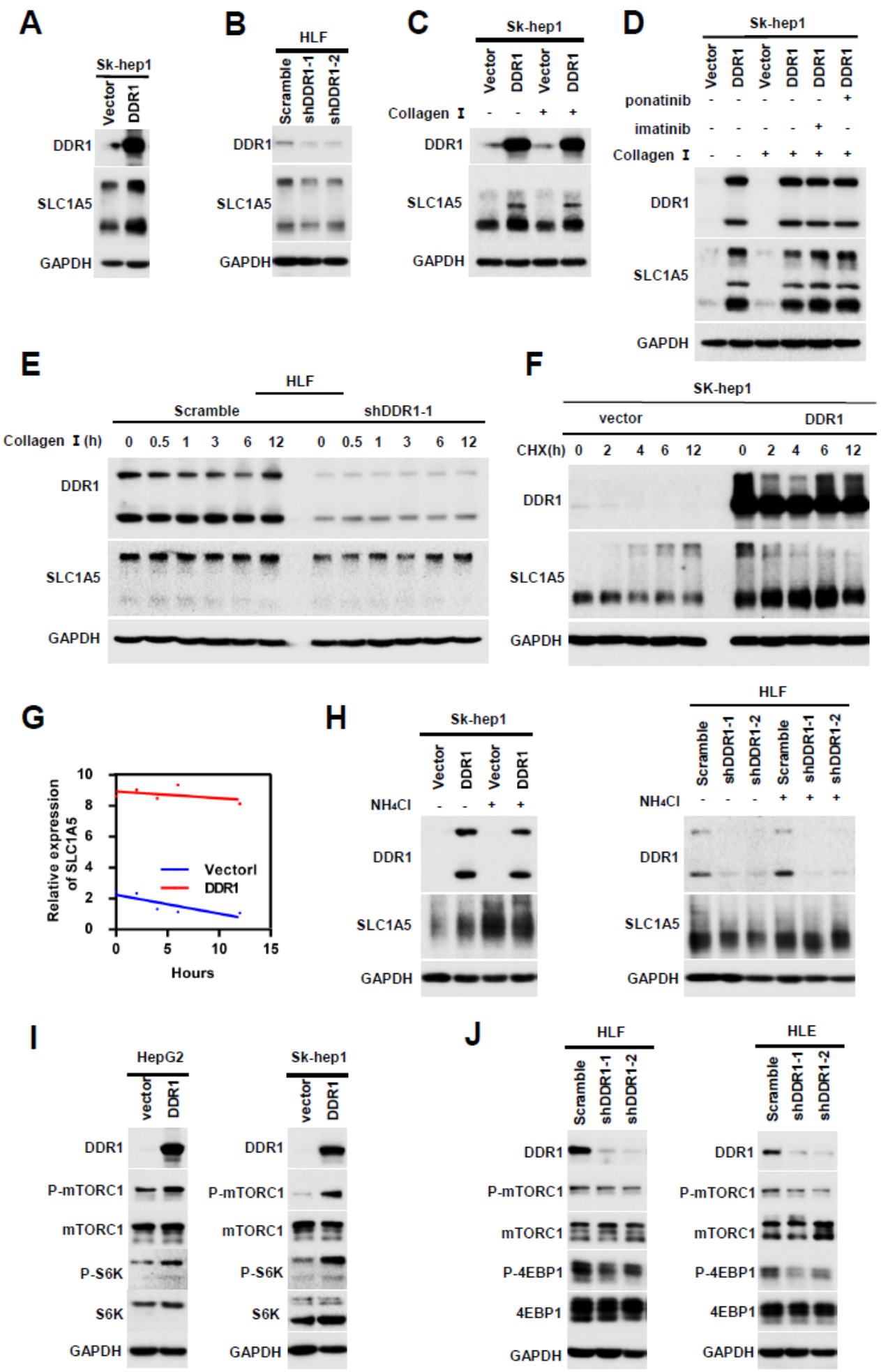

\section{Figure 4}

DDR1 stabilizes SLC1A5 through a kinase-independent manner $a, b$ The expression of SLC1A5 was detected by western blotting in indicated cells. c The expression of SLC1A5 was detected by western blotting in indicated cells with or without Collagen $₫$ stimulation(10ug/ml,6 hours).d DDR1-overexpression and control SK-hep 1 cells were pretreated with or without Collagen $\otimes(10 \mathrm{ug} / \mathrm{ml}, 3$ hours $)$ alone or in combination with ponatinib(100nM) and imatinib(100nM). The expression of SLC1A5 was detected by 


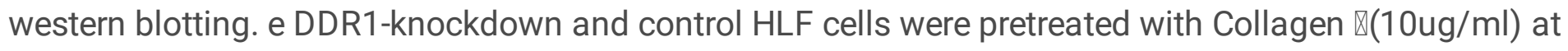
the indicated times. The expression of SLC1A5 was detected by western blotting. $\mathrm{f}, \mathrm{g}$ DDR1-

overexpression and control SK-hep 1 cells were pretreated with $\mathrm{CHX}(20 \mathrm{ug} / \mathrm{ml})$ at the indicated times. The expression of SLC1A5 was detected by western blotting. h DDR1-overexpression and control SK-hep1 cells were pretreated with NH4CL $(10 \mathrm{mmol} / \mathrm{L})$ for 6 hours. DDR1-knockdown and control HLF cells were pretreated with NH4CL $(10 \mathrm{mmol} / \mathrm{L})$ for 6 hours. The expression of SLC1A5 was detected by western blotting. i, j The expression of p-mTORC1, mTORC1, p-S6K, S6K, p-4EBP1 and 4EBP1 were detected by western blotting in indicated cells. 

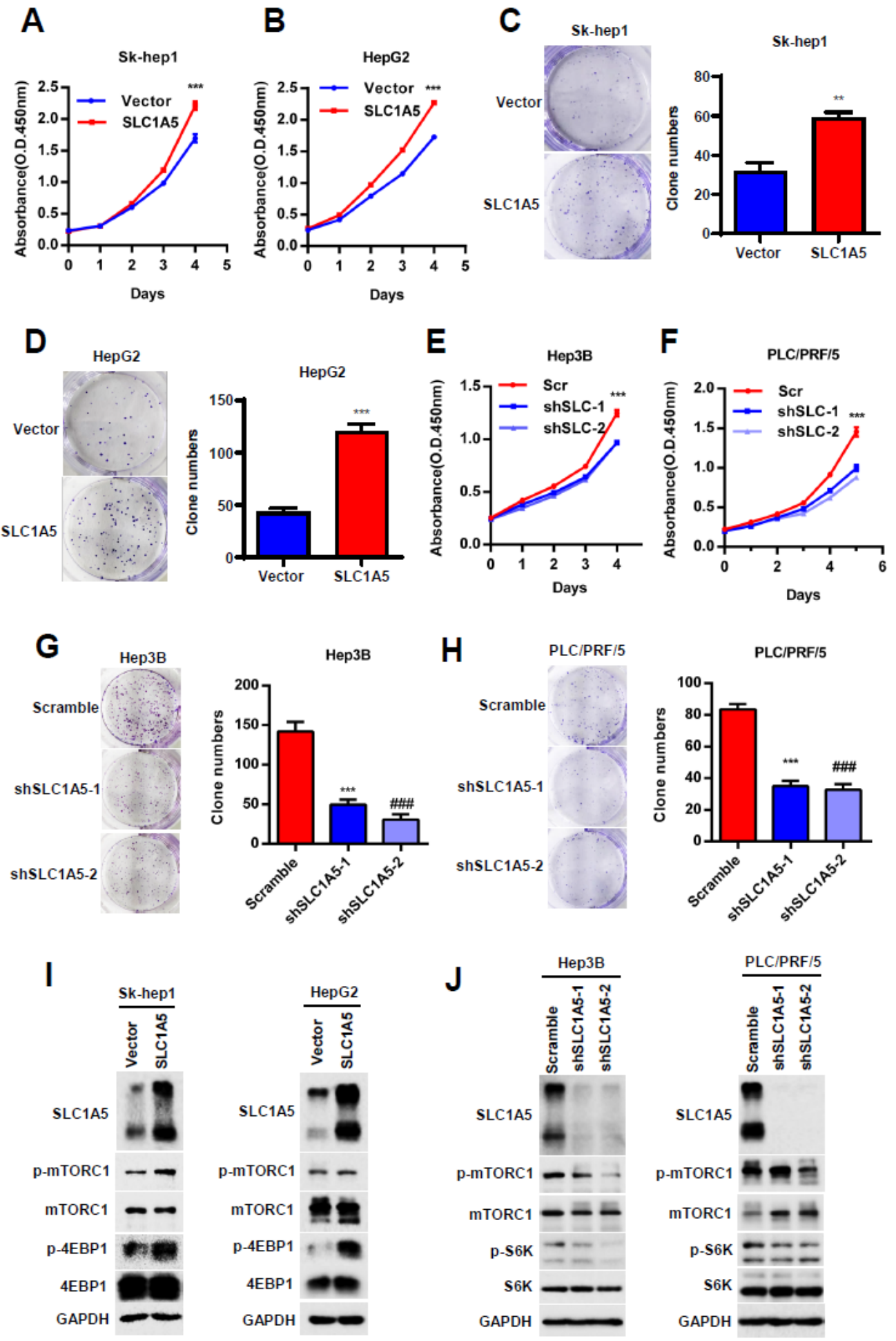

\section{Figure 5}

SLC1A5 promotes cellular proliferation, and mTORC1 signaling pathway in HCC cells. $a, b$ The effect of SLC1A5 overexpressing on SK-hep1 and HepG2 cell proliferation was assessed by the CCK8 assay. c, d The effect of SLC1A5 overexpression on SK-hep1 and HepG2 cell proliferation was assessed by a colonyformation assay. e, $f$ The effect of SLC1A5 knockdown on Hep3B and PLC cell proliferation was assessed by the CCK8 assay. $\mathrm{g}$, h The effect of SLC1A5 knockdown on Hep3B and PLC cell proliferation was 
assessed by a colony-formation assay. i, j The expression of p-mTORC1, mTORC1, p-S6K, S6K, p-4EBP1 and 4EBP1 were detected by western blotting in indicated cells.

A

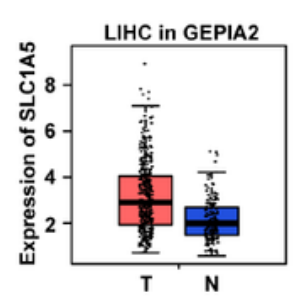

C

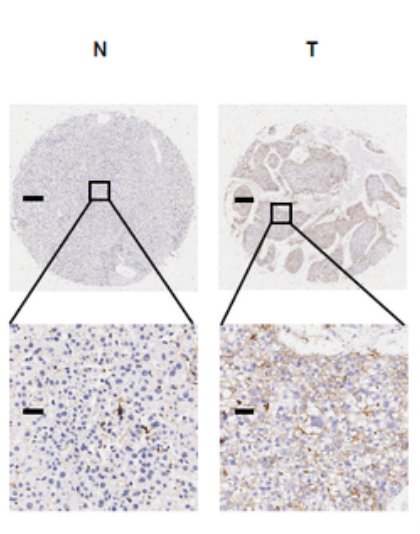

H
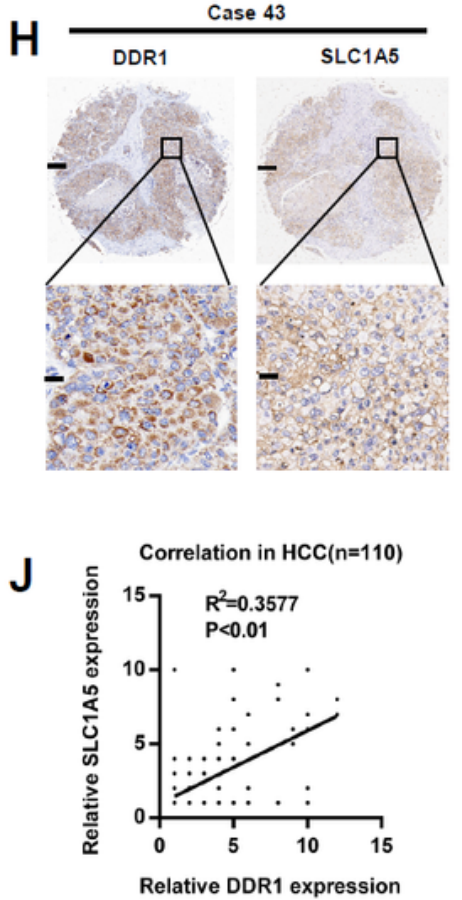

B

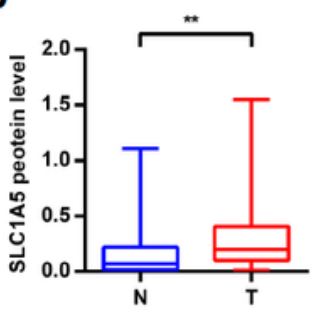

D E

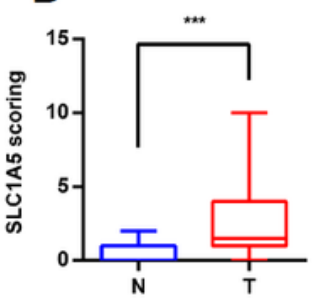

F SLC1A5 in TCGA LIHC

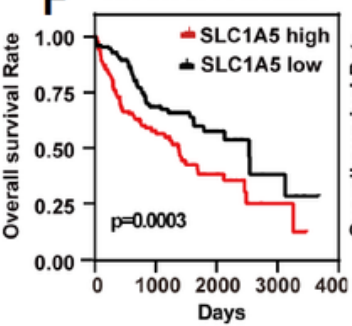

E Tongi cohort
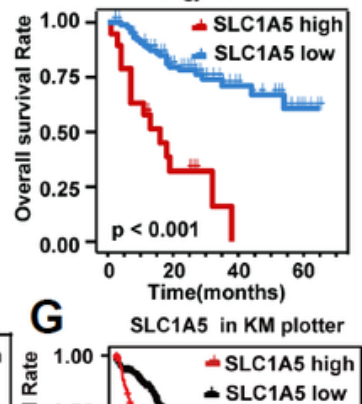

$\underline{\alpha}$ - 1 SLC1A5 low

密 0.50 -

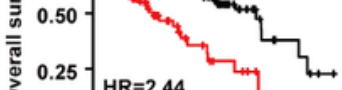

$H R=2.44$

p $<0.001$

Time(months)

I
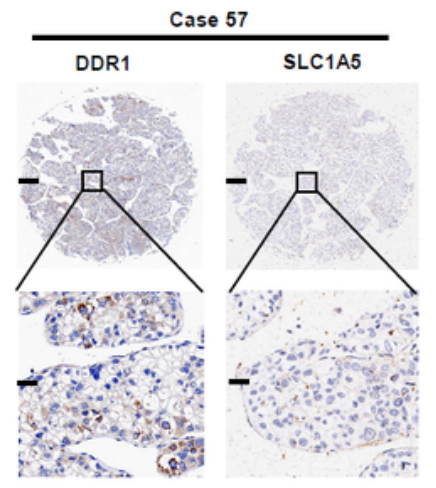

$\mathrm{K}$

Correlation in $\mathrm{HCC}(\mathrm{n}=43)$

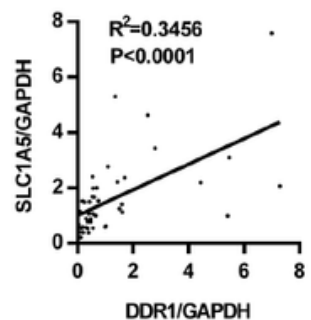

Figure 6

SLC1A5 is highly expressed in liver cancer and is positively correlated with DDR1. a The expression of SLC1A5 in HCC tissues $(\mathrm{T})$ compared with corresponding noncancerous liver tissues $(\mathrm{N})$ was analyzed using data sets from GEPIA2. b The protein level of SLC1A5 was examined in 62 pairs of HCC tumor 
tissues $(\mathrm{T})$ and corresponding peritumor tissues $(\mathrm{N})$ by western blot using anti-SLC1A5 antibody, where GAPDH was used as a loading control. The relevant density of SLC1A5 protein was analyzed and is shown. c, d Two representative immunohistochemical stains on a tissue array containing $110 \mathrm{HCC}$ samples with anti SLC1A5 antibody are shown. The average integrated optical density (IOD) of positive areas was analyzed. Scale bar represents $250 \mu \mathrm{m}$ (up), $25 \mu \mathrm{m}$ (down). e Overall survival analysis of HCC patients with SLC1A5 expression in 110 samples. f Overall survival analysis of HCC patients with SLC1A5 expression in TCGA data set $\mathrm{g}$ Overall survival analysis of HCC patients with SLC1A5 expression in KMplotter. h, I Representative immunohistochemical images showing positive correlation between DDR1 and SLC1A5 levels in human HCC samples. Scale bar represents $250 \mu \mathrm{m}$ (up), $25 \mu \mathrm{m}$ (down). j Correlation between DDR1 and SLC1A5 expression in tumor tissues by immunohistochemical stains assay. $\mathrm{k}$ Correlation between DDR1 and SLC1A5 expression in tumor tissues by western blotting assay.

\section{Supplementary Files}

This is a list of supplementary files associated with this preprint. Click to download.

- Figure1s.pdf

- Figure2s.pdf

- Figure3s.pdf

- Figure4s.pdf

- SupplementaryTable1.docx

- SupplementaryTable2.docx

- SupplementaryTable3.docx 